\title{
Generalized model of holographic recording in photopolymer materials
}

\author{
H.M. Karpov ${ }^{1}$, V.V. Obukhovsky ${ }^{1}$, T.N. Smirnova ${ }^{2}$ \\ ${ }^{1}$ Radiophysical Department, Kiev University, 60, Vladimirskaya St., 252033 Kiev,Ukraine \\ ${ }^{2}$ Institute of Physics of the NASU, 46, Prospekt Nauky, Kiev-22, Ukraine \\ (044)266-05-40; karpov@mail.univ.kiev.ua (H.M. Karpov) \\ (044)266-05-40;.vvo@rpd.univ.kiev.ua (V.V.Obukhovskv) \\ (044)265-50-72; smirnova@iop.kiev.ua (T.N. Smirnova)
}

\begin{abstract}
The generalized diffusion model of holographic recording in photopolymer materials has been offered. The theoretical description of hologram formation process is based on the concept of free volume redistribution and using the generalized diffusion equation. Free volume is produced in photopolymer medium due to the polymer shrinkage effect. The developed theory allows to take into account influence of inhomogeneous monomer distribution during recording process and shrinkage rate on a kinetics of a hologram formation. The principal influence of the diffusion/polymerization rates ratio on the hologram properties has been shown. The offered model allows also to describe relief formation process on the surface of a recording layer.
\end{abstract}

Keywords: photopolymer, holography, diffusion, shrinkage.

Paper received 26.07.99; revised manuscript received 30.09.99; accepted for publication 04.10.99.

\section{Introduction}

The quantitative theory of the holograms formation in photopolymer materials has been developed only during last 10 years [1-7]. The theoretical model under the consideration is based on the contemplation of two mutually connected processes: photopolymerization and diffusion. Photopolymerization occurs under the action of a inhomogeneous light field and is accompanied with the photoinduced diffusion redistribution of the photopolymer components. As a result of these processes the stable phase hologram is formed.

In the papers $[2,4]$ the consideration of matter transfer processes is based on standard diffusion equation. This method assumes the ability of free redistribution of the monomer to a homogeneous equilibrium state. However, the given assumption was not proved in the mentioned papers and its insufficient correctness is obvious by closer consideration.

Certainly, the presence of monomer concentration gradient, which occurs during the exposure of the medium in a inhomogeneous light field, is not a sufficient condition for the occurrence of the diffusion matter transfer. Under condition of total volume conservation, the monomer flow from dark to bright regions must be accompanied with the flow of substance in the opposite direction. This circumstance was not considered in the papers mentioned above.

In real photopolymer compositions (which we named «photoformers» [8]) the appearance of diffusion flows becomes possible due to polymer shrinkage effect [9-13] or due to the presence of an additional mobile component in the photosensitive medium [14-18].

The generalization of the diffusion equation for the case of diffusion redistribution of two mobile components (monomer and neutral component) with presence of the third motionless component (polymer) was carried out in [6].

In this paper the approach to the description of diffusion processes in photopolymer materials in presence of the shrinkage effect is considered on the basis of generalized diffusion equation obtained in [6].

\section{Polymer shrinkage effect and diffusion transfer of matter}

In this paper the shrinkage effect is considered as the formation of «free volume», which allows the monomer redistribution within medium bulk.

The main idea of our approach in the description of the diffusion process is the considering of the free volume as additional mobile component, which we designate as $X$. Thus, we have a model of the medium consisted of three components and two of them (monomer and $X$ component) are mobile. In this case the total volume of the system (with $X$ component) can be considered as the constant one. 


\section{H.M. Karpov et al.: Generalized model of holographic recording ...}

It is known that under the condition of total volume conservation there are $n-1$ independent gradients and $n-1$ independent flows in a $n$-component mixture. Taking into account an immovability of the polymer there are only one independent flow and two independent gradients in our case. The generalized diffusion equation for a similar media (binary photopolymer compositions) was obtained in our paper [6]. In this case the diffusion equation can be written down as follows

$$
\mathbf{j}_{M}=-\mathbf{j}_{X}=-D[X(\mathbf{r}, t) \nabla M(\mathbf{r}, t)-M(\mathbf{r}, t) \nabla X(\mathbf{r}, t)],
$$

where $M, \mathbf{j}_{M}, X, \mathbf{j}_{X}$ are volume fractions (normalized concentration) and flows of monomer and $X$ component accordingly; $D$ is diffusion coefficient. Note, that in a general case $D=D(M, P, \ldots)$ is a function of system state depending on coordinates and time.

In the phase of partial polymerization diffusion processes aspire to redistribute of monomer to the state, which is intermediate between homogeneous monomer distribution and homogeneous medium density. Thus, distributions $M^{e}(\mathbf{r}, t)$ and $X^{e}(\mathbf{r}, t)$, corresponding to an equilibrium state for fixed time $t$, satisfy the condition

$X^{e}(\mathbf{r}, t) / M^{e}(\mathbf{r}, t)=f(t)$,

where $f(t)$ does not depend on spatial coordinates. This condition corresponds to homogeneous monomer distribution throughout free volume formed as polymer shrinkage result. Note, that in general case equilibrium distribution of monomer $M^{e}(\mathbf{r}, t)$ is inhomogeneous due to the presence of the polymer.

It should be emphasized that the Eq. (1), in contrast to the Fick's diffusion equation, is nonlinear even in the case when diffusion coefficient $D$ is constant.

\section{General system of equations for inhomogeneous photopolymerization}

Basing on the model considered above, it is possible to write down the general system of equations for inhomogeneous polymerization of the photoformer.

We write down a conservation law of an arbitrary physical quantity $A$ in the standard form

$$
\frac{\partial A(\mathbf{r}, t)}{\partial t}+\nabla \mathbf{j}_{A}(\mathbf{r}, t)=S_{A}(\mathbf{r}, t)
$$

where $S_{A}(\mathbf{r}, t)$ is sources density of quantity $A$.

In general case the sources density function of monomer is

$S_{M}(\mathbf{r}, t)=-Q(\mathbf{r}, t) M(\mathbf{r}, t)$,

where $Q(\mathbf{r}, t)$ is local polymerization rate. Note that sources density of monomer is negative, that reflects its consumption in polymerization reaction.

Generally, a local polymerization rate is connected to distribution of irradiation in the interference pattern by nonlinear fashion. Besides, this connection is also nonlocal (at least, for time). This is proved, for example, by presence of postexposure self-amplification of the holograms in the photopolymer media [19].
Obtaining an explicit relationship between $Q(\mathbf{r}, t)$ and $I(\mathbf{r}, t)$ is a complicated problem which exceeds the bounds of this paper. Note only that the approaches of the solution of this problem can be based on the consideration of chemical reactions kinetics or on the analysis of physical kinetics of phase transformation processes. In the first case $Q(\mathbf{r}, t)$, to within coefficient, corresponds to distribution of polymer radicals concentration which can be connected to $I(\mathbf{r}, t)$ by the system of differential equations [6].

To obtain the equation for the density of polymer and $X$ component sources, we define shrinkage coefficient as follows:

$s=\frac{V_{M}-V_{P}}{V_{M}}$,

where $V_{M}, V_{P}$ are volumes of monomer and polymer, respectively.

Taking into account (5), the equations for the density of $X$ and $P$ components sources can be written down as follows:

$S_{X}=-s S_{M}, S_{P}=-(1-s) S_{M}$,

where $S_{M}$ is the monomer sources density determined by expression (4).

As the result, using the equations $(1,4,6)$, we can obtain the following system of equations which describes polymerization of the photoformer:

$$
\frac{\partial X(\mathbf{r}, t)}{\partial t}=s Q(\mathbf{r}, t) M(\mathbf{r}, t)+\nabla[D(M(\mathbf{r}, t) \nabla X(\mathbf{r}, t)-X(\mathbf{r}, t) \nabla M(\mathbf{r}, t))]
$$

$\frac{\partial M(\mathbf{r}, t)}{\partial t}=-Q(\mathbf{r}, t) M(\mathbf{r}, t)+\nabla[D(X(\mathbf{r}, t) \nabla M(\mathbf{r}, t)-M(\mathbf{r}, t) \nabla X(\mathbf{r}, t))]$,

$\frac{\partial P(\mathbf{r}, t)}{\partial t}=(1-s) Q(\mathbf{r}, t) M(\mathbf{r}, t)$.

The system (7) has to be complemented with the initial conditions:

$M(\mathbf{r}, t=0)=1, X(\mathbf{r}, t=0)=0, P(\mathbf{r}, t=0)=0$.

If the volume fractions of the initiator and intermediate products of polymerization reaction are small (that is a typical situation), the law of total volume conservation is

$M(\mathbf{r}, t)+P(\mathbf{r}, t)+X(\mathbf{r}, t)=1$.

In turn, the expression (9) allows excluding one equation from the system (7).

\section{Model analysis and discussion of results}

The analysis of the developed model of holographic recording was carried out by the numerical solution of the system (7) for the case of recording of the transmitting hologram grating in the interference pattern of two laser beams. In the simplest case the spatial distribution of irradiance in the recording layer is

$I(x)=I_{0}[1+m \cos (2 \pi x / \Lambda)]$ 


\section{H.M. Karpov et al.: Generalized model of holographic recording ...}

where $I_{0}=I_{1}+I_{2}$ is the total intensity of recording beams; $m=2 \sqrt{I_{1} I_{2}} /\left(I_{1}+I_{2}\right)$ is the fringe visibility; $\Lambda$ is the fringe spacing (the $x$ axis is directed along recording layer surface).

Comparability with results of papers $[13,14]$ was achieved by taking local polymerization rate function as follows

$$
Q(x, t)=k_{p} I^{1 / 2}(x) .
$$

As it follows from [7] this form of $Q(x, t)$ can be considered as zero approach for compositions with the radical polymerization mechanism. The representation $Q(x, t)$ as $(11)$ does not allow describing some important effects (for example, postexposure self-amplification of the holograms), however, it allows us to be concentrated on the most essential aspects of holograms formation in the photopolymers.

For the convenience of the further analysis we rewrite system (7) using dimensionless coordinates with excluding one of the equations accordingly to (9):

$$
\begin{aligned}
& \frac{\partial X\left(x^{\prime}, t^{\prime}\right)}{\partial t^{\prime}}=s \mathrm{f}\left(x^{\prime}\right) M\left(x^{\prime}, t^{\prime}\right)+ \\
& +D_{e f}\left[M\left(x^{\prime}, t^{\prime}\right) \frac{\partial^{2} X\left(x^{\prime}, t^{\prime}\right)}{\partial x^{\prime 2}}-X\left(x^{\prime}, t^{\prime}\right) \frac{\partial^{2} M\left(x^{\prime}, t^{\prime}\right)}{\partial x^{\prime 2}}\right], \\
& \frac{\partial P\left(x^{\prime}, t^{\prime}\right)}{\partial t^{\prime}}=(1-s) \mathrm{f}\left(x^{\prime}\right) M\left(x^{\prime}, t^{\prime}\right) .
\end{aligned}
$$

Here $x^{\prime}=x / \Lambda, t^{\prime}=t / \tau_{p}$ are dimensionless coordinate and time; $\tau_{p}=\left(k_{p} I_{0}^{1 / 2}\right)^{-1}$ is characteristic time of polymerization process; $f\left(x^{\prime}\right)=1+m \cos \left(2 \pi x^{\prime}\right) ; D_{e f}=\tau_{p} D_{0} / \Lambda^{2}$ is effective diffusion coefficient. In the system (12) we also assume that the diffusion coefficient during the recording process is constant and equal to $D_{0}$.

Diffraction properties of the holographic grating are determined by the amplitudes of spatial harmonics of refractive index modulation and can be computed, for example, by using coupled wave theory which includes these amplitudes as parameters. Therefore, for the description of diffraction properties of the grating, we will use amplitudes of spatial harmonics of component volume fraction modulation which are determined by expression

$$
Y_{i}(t)=\frac{2}{\Lambda} \int_{-\Lambda / 2}^{\Lambda / 2} Y(x, t) \cos (2 \pi i x / \Lambda) d x
$$

where $i=1,2, \ldots$ and $Y$ can be $M, P$ and $X$. Further we will designate amplitude of the $i$-th harmonic by index «i» in corresponding symbols.

The numerical experiments have shown the principal influence of effective diffusion coefficient value on hologram formation process. This quantity, effecctively, is ratio of the characteristic polymerization time to the monomer diffusion time at a distance of the $\Lambda$ order.

The typical kinetics of the first harmonic of spatial components distribution during recording process shown in Fig.1.
These curves are obtained by the numerical solution of the system (12) with $s=0.2, m=0.98$ for different values of $D_{e f}$. As follows from this figure, for any values of effective diffusion coefficient there is a significant gradient of monomer concentration during the recording process. This conclusion differs essentially from the results obtained in [4].

Certainly, if diffusion process proceeds much faster than polymerization process (in our case this corresponds to $D_{e f}>>1$ ), in each specific time moment the system is in the state close to equilibrium from the point of view of diffusion matter transfer. Used in [4] the standard diffusion equation means uniformity of the monomer equilibrium state, from that the conclusion about absence of an significant monomer concentration gradient during recording follows.

As the optical properties of the monomer and polymer are different, the monomer distribution influences on modulation of optical properties of recording layer in a direct way. Possibility of account of monomer distribution heterogeneity allows describing hologram kinetics more accurately as compared with the approach used in $[2,4]$.

A typical dry photopolymer system consists of a monomer, polymeric binder and photoinitiator. In this case, the main result of recording process is spatial modulation of density of polymer and its optical properties. With absence of a polymeric binder (or its insufficient rigidity), however, there are conditions for formation of the relief on the layer surface during recording process.

The discussed theoretical model, in contrast to earlier offered models, allows describing formation of surface relief in a natural way. This process can be considered as a «displacement» of the $X$ component (free volume) onto the recording layer surface. There is possibility to analyze the surface relief formation by considering the $X$ component redistribution kinetics during holographic recording.

For example, as follows from Fig.1, for small values of effective diffusion coefficient $D_{e f}$, the sign of the first harmonic of $X$ component distribution changes during recording. In a context of surface relief consideration, it means that at initial stage of hologram formation the hollows of the relief correspond to bright regions, and after recording completion they correspond to dark regions.

The explicit accounting of shrinkage effect allows also investigating dependence of holographic recording efficiency

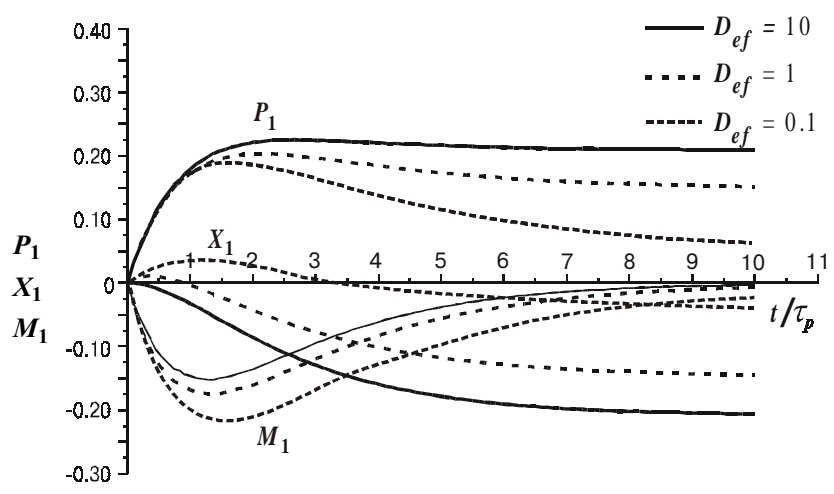

Fig.1. Kinetics of the first harmonic of spatial components distribution during recording process for different values of $D_{e f}$. 


\section{H.M. Karpov et al.: Generalized model of holographic recording ...}

(steady state value of the first spatial harmonic of polymer distribution $P_{1}^{s t}$ ) on shrinkage rate. This dependence with various values $D_{e f}$ is represented on Fig.2. As follows from this figure, the optimal value of shrinkage coefficient exists. It corresponds to the peak recording efficiency.

The theoretical dependencies of the first three harmonics of steady state polymer volumetric part distribution $P^{s t}\left(x^{\prime}\right)$ from $D_{e f}$ are shown in Fig.3. As follows from this figure, over the range $0<D_{e f}<0.1$ the hologram is characterized by low efficiency and large relative amplitude of higher $(i>1)$ harmonics, which is an exidence of a strong nonlinearity of the recording process. Efficiency and linearity of recording rise considerably with increasing $D_{e f}$ and reach saturation at $D_{e f} \cong 1$. The specified dependencies qualitatively agree with the results obtained in papers $[4,7]$.

Fig.4 shows the profiles of steady state polymer distribution $P^{s t}$ for different $D_{e f}$ values. Under small $D_{e f}$ values the profile of $P^{s t}$ strongly differs from the irradiation distribution and has characteristic two-peak shape.

Thus, $D_{e f}$ can be considered as criterion of holographic recording efficiency. The condition of obtaining of effective recording can be written as $D_{e f}>1$. As follows from the definition of effective diffusion coefficient $D_{e f}$ its value decreases with increase of grating period and, hence, the diffusion process rate limits material performance over high spacing range.

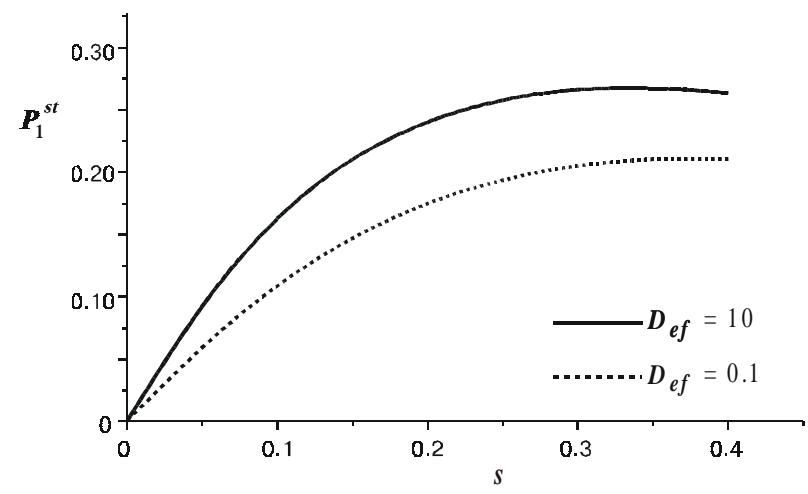

Fig.2. Dependencies of steady state value of the first spatial harmonic of polymer distribution on shrinkage rate for different values of $D_{e f}$.

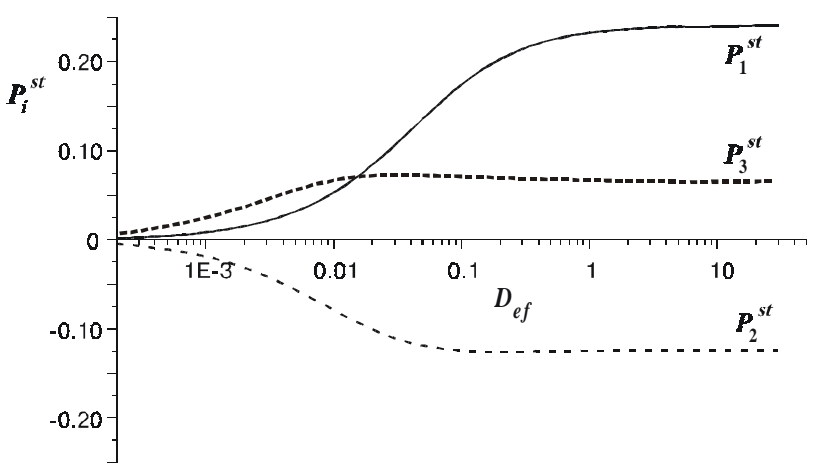

Fig.3. Dependencies of the first three harmonics of steady state polymer distribution on $D_{e f}$.

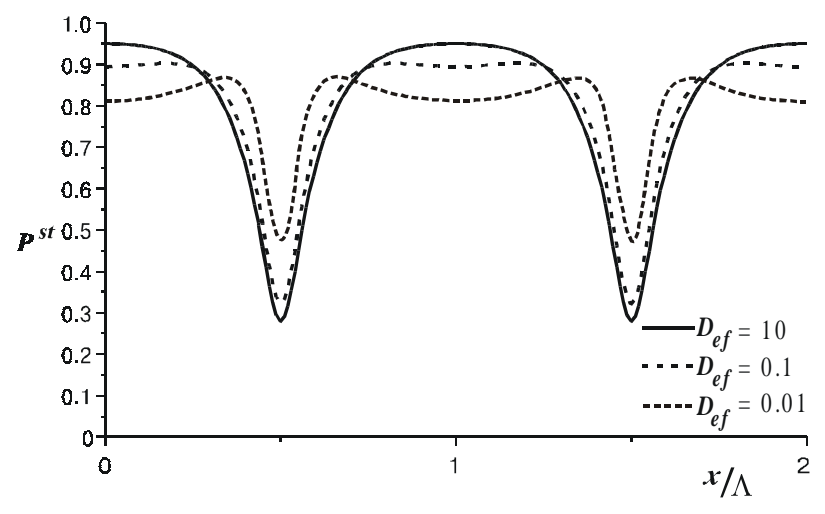

Fig.4. Profiles of steady state polymer distribution for different values of $D_{e f}$.

\section{Conclusions}

The generalized diffusion model of hologram formation in photopolymer materials has been offered. The model is based on using the nonlinear diffusion equation for the description of the matter transfer processes in presence of the polymer shrinkage effect.

The obtained results allow taking into account influence of monomer distribution heterogeneity during recording on hologram diffraction efficiency kinetics. The model also allows analyzing the influence of shrinkage rate on holographic recording efficiency.

The analysis of the theoretical model has shown the principal influence of the diffusion/polymerization rates ratio on hologram properties.

Using the offered model allows also t $j$ describe the surface relief formation process which can take place during hologram recording.

\section{References}

1. E.S. Gyulnazarov, V.V. Obukhovskii, T.N. Smirnova, Theory of holographic recording on a photopolymerized material // Optics and Spectroscopy 69(1), pp.109-111 (1990).

2. R.R. Adhami, D.J. Lanteigne and D.A. Gregory, Photopolymer hologram formation theory // Microwave and Opt. Tech. Let. 4(3), pp.106109 (1991).

3. V.V. Obukhovskii, T.N. Smirnova, Model of holographic recording on photopolymerizing composites // Optics and Spectroscopy 74(4), pp.462-466 (1993).

4. G.Zhao and P. J. Mouroulis, Diffusion model of hologram formation in dry photopolymer materials // Modern Opt. 41(10), pp.1929-1939 (1994).

5. G.Zhao and P. J. Mouroulis, Extension of a diffusion model for holographic photopolymers // Modern Opt. 42(12), pp.2571-2573 (1995).

6. G.M. Karpov, V.V. Obukhovskii, T.N. Smirnova, Theory of hologram formation in photopolymer materials with a polymerization diffusive recording mechanism. I. General equations system // Optics and Spectroscopy 81(6), c.1033-1038 (1996).

7. G.M. Karpov, V.V. Obukhovskii, T.N. Smirnova, T.A. Sarbaev, Theory of hologram formation in photopolymer materials with a polymerization diffusive recording mechanism. II. Regularities of the process and criterion of holographic recording efficiency // Optics and Spectroscopy 82(1), pp.131-137 (1997).

8. G.M. Karpov, V.V. Obukhovsky, T.N. Smirnova, Photoformers: materials for holographic recording // Proc. SPIE. 2795, pp.294-305 (1996). 


\section{H.M. Karpov et al.: Generalized model of holographic recording ...}

9. W.S.Colburn and K.A. Haines, Volume hologram formation in photopolymer materials // Appl. Opt. 10(7), pp.1636-1641 (1971).

10. R.H. Wopshall and T.R. Pampalone, Dry photopolymer films for recording holograms // Appl.Opt. 11(9), pp.2096-2105 (1972).

11. B.L.Booth, Photopolymer material for holography // Appl.Opt. 14(3), pp.593-601 (1975)

12. D.H. Whitney and R.T. Ingwall, The fabrication and properties of composite holograms recorded in DMP-128 photopolymer // Proc. SPIE 1213, pp.18-26 (1990).

13. W.K. Smothers, B.M. Monroe, A.M. Weber and D.E. Keys, Photopolymers for holography // Proc. SPIE. 1212, pp.20-29 (1990).

14. W.J. Tomlinson, E.A. Chandross, H.P. Weber, G.D. Aumiller, Multicomponent photopolymer systems for volume phase holograms and grating devices // Appl. Opt. 15(2), pp.534-541 (1976).

15. T.N. Smirnova, E.S. Gyulnazarov, E.A. Tikhonov, Optical nonlinearity and holographic recording of stable periodic structures in polymeric photorefractive media // Proc. SPIE 1017, pp.190-192 (1988)

16. M. Kawabata, A. Sato, I. Sumiyoshi and T. Kubata, Photopolymer system and its application to a color hologram // Appl. Opt. 33(11), pp.2152-2156 (1994)

17. E.S. Gyulnazarov, T.N. Smirnova, E.A. Tikhonov, Post-polimerization self-amplification of holohrams in photopolimer compositions // Journal of Technical Physics. 61(1), c.111-117 (1991) (rus). 